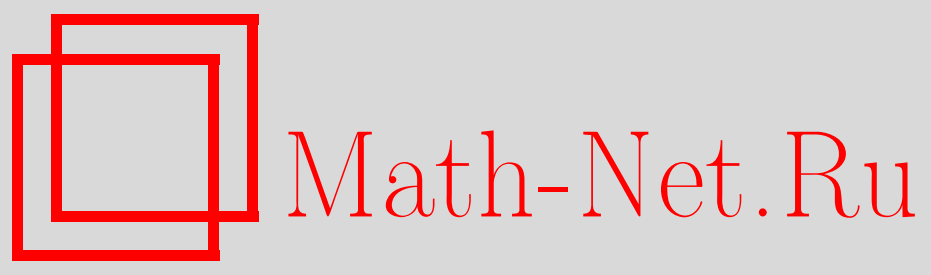

Н. Э. Добринская, Конфигурационные пространства с метками и пространства петель на $K$-произведениях, УМH, 2008, том 63, выпуск 6, 161-162

DOI: https://doi.org/10.4213/rm9256

Использование Общероссийского математического портала Math-Net.Ru подразумевает, что вы прочитали и согласны с пользовательским соглашением http://www . mathnet.ru/rus/agreement

Параметры загрузки:

IP : 54.237 .59 .107

26 апреля 2023 г., 17:06:05

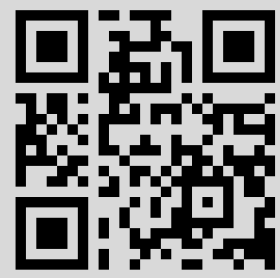




\section{Конфигурационные пространства с метками и пространства петель на $K$-произведениях}

\section{Н. Э. Добринская}

Пусть $K=\{\sigma \subset[m]\}$ - симплициальный комплекс на множестве вершин $[m]=$ $\{1,2, \ldots, m\}$, а $\mathbf{X}=\left(X_{1}, \ldots, X_{m}\right)$ - последовательность пространств с отмеченной точкой. Полиэдральным произведением $\mathbf{X}^{K}$, или $K$-произведением, называется подпространство прямого произведения

$$
\mathbf{X}^{K}=\bigcup_{\sigma \in K} \mathbf{X}^{\sigma} \subset \prod_{i \in[m]} X_{i},
$$

где $\mathbf{X}^{\sigma} \subset \prod X_{i}$ и вложение $\mathbf{X}^{\sigma} \subset \prod X_{i}$ задается при помощи отмеченных точек.

Конструкция пространства $\mathbf{X}^{K}$ впервые появилась еще в работе [1] как обобщение обычных букетов ( $K$ - несвязное объединение вершин), декартовых произведений $(K-$ симплекс), жирного букета $(K$ - граница симплекса), но особый интерес к ней возник благодаря торической топологии, где она играет важную роль. Так, например, задача о гомотопическом типе торических многообразий привела к изучению пространства петель на $K$-произведениях [2].

Данная работа посвящена вычислению алгебры гомологий пространства петель на $K$-произведении при наличии достаточной информации о пространствах $X_{i}$. Полученные результаты открыли удивительные связи этого вопроса с другими задачами о наборах подпространств в евклидовых пространствах, конфигурационных пространствах частиц с метками, а также с вопросами коммутативной алгебры.

Для произвольного $k \in \mathbb{N}_{0}$ из множества неотрицательных целых чисел обозначим через $B(k)$ конфигурационное пространство неупорядоченных наборов $k$ точек на вещественной прямой:

$$
B(k)=\{\mathbf{t}=\{t(1), \ldots, t(k)\} \subset \mathbb{R} \mid t(i) \neq t(j), i \neq j\} .
$$

Построим пространство $C_{K}=\bigsqcup_{I \in \mathbb{N}_{0}^{m}} C_{K}(I)$, где для каждого $I=\left\{i_{1}, \ldots, i_{m}\right\}$ компонента $C_{K}(I) \subset B\left(i_{1}\right) \times \cdots \times B\left(i_{m}\right)$ определяется следующим условием:

$$
\left(\mathbf{t}_{1}, \ldots, \mathbf{t}_{m}\right) \in C_{K}(I) \Longleftrightarrow \bigcap_{j \in \tau} \mathbf{t}_{j}=\varnothing \quad \text { для любого } \tau \notin K .
$$

Отметим, что все $C_{K}(I)$ гомеоморфны либо дополнениям к конфигурациям диагональных подпространств, ассоциированных с $K$, либо некоторым их компонентам.

Назовем индекс из $[m]$ цветом частицы. Частицы каждого цвета естественным образом упорядочены на прямой. Таким образом, все частицы индексируются парами $(j, k)$, где где $j \in[m]$ и $k \in\left[i_{j}\right]$.

Рассмотрим операцию удвоения частицы в $C_{K}$ - для некоторого малого $\varepsilon$ мы заменяем ее на две частицы того же цвета на расстоянии $\varepsilon$ и с центром тяжести в координате удаленной частицы. Эта процедура индуцирует операции в гомологиях: $\mu_{j, k}: H_{*}\left(C_{K}(I)\right) \rightarrow H_{*}\left(C_{K}\left(I+e_{j}\right)\right)$, где $j \in[m], k \in\left[i_{j}\right]$, а $e_{j}$ обозначает последовательность $\{0\}^{j-1} \times\{1\} \times\{0\}^{m-j}$.

Существует гомотопическая деформация пространства $C_{K}$, на которой можно ввести структуру моноида. Следовательно, $H_{*}\left(C_{K}\right)$ является алгеброй.

Гомологии всех пространств будем рассматривать над фиксированным полем $R$.

Теорема 1. Пусть $X_{1}, \ldots, X_{m}$ - односвязные пространства с отмеченной точкой. Тогда имеет место изоморфизм градуированных алгебр

$$
H_{*}\left(\Omega\left(\mathbf{X}^{K}\right)\right) \cong \bigoplus_{I \in \mathbb{N}_{0}^{m}} H_{*}\left(C_{K}(I)\right) \otimes \widetilde{H}_{*}(\Omega \mathbf{X})^{\otimes I} / \sim
$$


Отношение эквивалентности задается следующими условиями:

$$
\left(\mu_{j, k} y\right)\left(\cdots \otimes x_{j}^{k} \otimes x_{j}^{k+1} \otimes \cdots\right) \sim y\left(\cdots \otimes x_{j}^{k} \cdot x_{j}^{k+1} \otimes \cdots\right) .
$$

Комплекс называется флаговым, если любой набор вершин $\tau \in[m]$, попарно соединенных ребрами (т. е. $\{i, j\} \in K$ для любых $i, j \in K$ ), содержится в $K: \tau \in K$. Из теоремы 1 мы получаем следующее утверждение.

СлеДСтвиЕ 2. Для любого флагового симплициального комплекса $K$ имеет место следуюший изоморфизм алгебр:

$$
H_{*}\left(\Omega \mathbf{X}^{K}\right) \cong \bigsqcup_{i=1}^{m} H_{*}\left(\Omega X_{i}\right) / \sim,
$$

где $x \cdot y \sim(-1)^{\operatorname{deg} x \operatorname{deg} y} y \cdot x$ для всех $x \in H_{*}\left(\Omega X_{i}\right), y \in H_{*}\left(\Omega X_{j}\right) u\{i, j\} \in K$.

Это следствие было известно в частных случаях, когда $X_{i} \cong \mathbb{C P}^{\infty}$ для всех $i$ (см. [3]) или $X_{i} \cong S^{2 n+1}$ для всех $i$ (см. [4]).

Введем мультиградуированную алгебру $\wedge(K)=\wedge\left[v_{1}, \ldots, v_{m}\right] / I_{\mathrm{SR}}$, где $\operatorname{deg} v_{i}=1$, a $I_{\mathrm{SR}}$ - идеал Стенли-Райснера, порожденный мономами вида

$$
v_{I}:=v_{i_{1}} \cdots v_{i_{s}}=0 \text { для } I=\left\{i_{1}, \ldots, i_{s}\right\} \notin K .
$$

Теорема 3. Алгебра $H_{*}\left(C_{K}\right)$ изоморфна гомологиям модели Адамса-Хилтона, построенной по стандартному клеточному разбиению пространства $\left(S^{1}, \ldots, S^{1}\right)^{K}$. Имеет место следующий мультиградуированный изоморфизм алгебр:

$$
H_{*, *}\left(C_{K}\right) \cong \operatorname{Ext}_{*, *}^{\wedge(K)}(R ; R) .
$$

Дадим геометрическое объяснение полученных результатов. Существует геометрическая модель для пространства петель $\Omega\left(\mathbf{X}^{K}\right)$ в виде конфигурационного пространства $C_{K}$ с метками в $\Omega X_{i}$ и возможными слияниями частиц (подробности этой теории см. в [5]). В частном случае, когда каждое из пространств в $\mathbf{X}$ является надстройкой: $X_{i} \simeq \Sigma Y_{i}$ для некоторого связного $Y_{i}$, модель упрощается:

$$
\Omega(\Sigma \mathbf{Y})^{K} \simeq \bigsqcup C_{K}(I) \times \mathbf{Y}^{I} / \sim
$$

где отношение эквивалентности определяется условием, что если метка частицы отмеченная точка из $Y_{i}$, то частица исчезает.

В этом случае $H_{*}\left(\Omega \Sigma Y_{i}\right)$ изоморфна свободной тензорной алгебре $T\left(\widetilde{H}_{*}(Y)\right)$, и изоморфизм теоремы 1 становится расщеплением:

$$
H_{*}\left(\Omega(\Sigma \mathbf{Y})^{K}\right) \cong \bigoplus_{I \in \mathbb{N}_{0}^{m}} H_{*}\left(C_{K}(I)\right) \otimes \widetilde{H}_{*}(\mathbf{Y})^{\otimes I} .
$$

Возникает вопрос, является ли это расщепление геометрическим.

Теорема 4. Пусть $X_{i} \simeq \Sigma Y_{i}, i \in[m]$, где $Y_{i}$ - связные пространства. Тогда существует стабильная гомотопическая эквивалентность

$$
\Omega(\Sigma \mathbf{Y})^{K} \simeq_{\mathrm{s}} \bigvee_{I \in \mathbb{N}_{0}^{m}} C_{K}(I)_{+} \wedge \mathbf{Y}^{\wedge I}
$$

\section{Список литературы}

[1] D. Anick, Lecture Notes in Math., 1051 (1984), 331-350. [2] В. М. Бухштабер, Т. Е. Панов, Торические действия в топологии и комбинаторике, МЦНМО, М., 2004. [3] Т. Е. Panov, R. Ray, Contemp. Math., 460 (2008), 293-322. [4] S. Papadima, A. Suciu, Math. Ann., 334:3 (2006), 533-555. [5] Н. Э. Добринская, Тр. МИАН, 252 (2006), 37-54.

Н. Э. Добринская (N. È. Dobrinskaya)

Vrije Universiteit Amsterdam

E-mail: NE.Dobrinskaya@few.vu.nl
Представлено В. М. Бухштабером Принято редколлегией 10.11.2008 